\title{
Hoechst Adverse Reaction Terminology System
}

National Cancer Institute

\section{Source}

National Cancer Institute. Hoechst Adverse Reaction Terminology System. NCI

Thesaurus. Code C71884.

A terminology for coding of adverse reactions, developed by Hoechst in 1991. 